\title{
New directions to understand and learn from embryonic diapause in mammals
}

\author{
P Comizzoli \\ Smithsonian National Zoo \& Conservation Biology Institute, NW Washington DC, USA
}

Corresponding author email: comizzolip@si.edu

\begin{abstract}
Embryonic diapause is a key strategy to extend pregnancy until conditions are ideal for birth and postnatal survival. There is still a lot to discover about this unique phenomenon observed in more than 130 mammalian species. The present review aims at complementing existing research efforts by (1) identifying new directions for a better understanding of embryonic diapause in mammals and (2) considering this complex mechanism as a source of inspiration for other areas in cellular biology. Comparative explorations in different species of new molecular players associated with the use of emerging technologies in the study of embryos (epigenetics for instance), the uterus (immune cells, microbiota, cell-free DNA), and the whole organism (remote sensing, systems biology) will shed some new lights on embryonic diapause characterizations. Collective results of advanced studies should be integrated into the measurement of climate and environmental changes potentially influencing the physiology of females and their arrested embryos. Interestingly, lessons from nonmammalian species using similar strategies (killifish for instance) could also improve our understanding of this unique phenomenon. Furthermore, studying embryonic diapause offers a great opportunity to decipher other cellular mechanisms and develop new applications (stem cell technologies, cancer treatments, contraceptive methods, short-term storage of embryos, or early fetal loss prevention). Overall, these new ideas and directions should define some themes for a future International Symposium on Embryonic Diapause.
\end{abstract}

\section{Introduction}

Similar to delayed fertilization (sperm storage in the female reproductive tract) or delayed development after embryo implantation, embryonic diapause is a key strategy to extend pregnancy until conditions are ideal for birth and postnatal survival [1]. Since its first observation in roe deer in the $19^{\text {th }}$ century, embryonic diapause has been described in more than 130 mammalian species and involves very diverse physiological and cellular mechanisms [1]. Within embryonic diapause, five different conditions of the blastocyst can be recognized: 1) no growth in unilaminar blastocysts with a zona pellucida (kangaroos); 2 ) no growth in unilaminar blastocysts without a zona pellucida (mice and rats); 3) slow growth in unilaminar blastocysts with a zona pellucida (mustelids, bears, seals); 4) slow growth in bilaminar blastocysts without a zona pellucida (roe deer); and 5) implanted but undifferentiated (bats). Current knowledge of embryonic diapause allows to distinguish two main types - lactational versus seasonal controlled diapause [2]. The 3rd International Symposium on Embryonic Diapause organized in June 2019 was a unique opportunity to check the state-of-the-art in that complex biological mechanism (see Indroduction this volume). Some presentations also highlighted new approaches and tools that could help to decipher some of the remaining mysteries. However, there are still many questions 
to be answered as well as more species to be thoroughly studied. Research efforts have to concentrate on (1) embryology during diapause while embryogenesis is either suspended or slowed down (2) structure and function of uterus during diapause and (3) embryo-uterus interactions during onset of diapause, diapause itself, and resumption of embryo development/implantation. Importantly, those three components highly depend on the physiology of the whole organism. Comparative explorations of new molecular players in different species associated with the use of cutting-edge approaches are necessary to make progress. The main objective of this review is to propose new research directions, in addition or in complement to the existing one, for better understanding this unique phenomenon. Studying diapause also provides new insights not only into mammalian reproduction evolution but has potential applications to general cellular biology and for the development of new treatments or technologies. This will be reviewed in a second part.

\section{Looking for new players involved in embryonic diapause}

\section{More micro-RNA explorations}

Micro-RNAs regulate gene expression in different physiological systems. While micro-RNAs of the Let-7 family have been previously shown to be potentially regulating diapause, additional microRNA regulators of diapause and their target genes still remain to be explored [3]. Interestingly, consistent connections between 38 microRNA and 274 target genes were identified for up or down regulations in the mouse model. Specifically, microRNA target genes showed more enrichment in stress response, hypoxia, and hormone response pathways, which is consistent with the diapause phenotype. Inversely, target genes down-regulated in diapause (their microRNAs being up-regulated in diapause) showed enrichment in cellular morphogenesis, differentiation, and development, which is consistent with diapause as a suspended developmental stage [3].

\section{Detection of new polyamines}

Compounds like polyamine have already been shown to regulate carnivore embryonic diapause [4]. Recent studies in the fruit bat (Cynopterus sphinx) also demonstrated that putrescine plays two separate roles during embryonic diapause (supporting corpus luteum and basal synthesis of progesterone in the ovary and inhibition of cell proliferation in the utero-embryonic unit) [5]. In addition to the detection of additional compounds, their excretion could also be non-invasively monitored (in fecal or urine samples for instance) to fully characterize the role of those molecules in embryonic diapause.

Role of bone marrow derived immune cells and preferred localizations of implantation in the uterus

It is well established that uterine implantation sites are areas of infiltration of many bone marrow derived immune cells, which play important roles at the maternal-fetal interface to promote successful pregnancy [6]. A new study provided evidence that those cells have a nonhematopoietic physiologic contribution to the decidual stroma and play an important role in implantation and pregnancy maintenance [7]. Importantly, nonhematopoietic immune cells can influence the decidual molecular milieu and overcome implantation defects. While more links have to be established with delayed implantation, these new data raise the question about possible regulations by bone marrow derived immune cells of the timing of implantation [7]. This would also help to determine, in different species, if there is a specific region in the uterus for implantation after embryonic diapause. 


\section{Characterization of microbiota in the reproductive tract}

Communities of microbes have coevolved in animal organisms and are found in almost every part of the body. Compositions of those communities (microbiota) as well as their genomes and genes (microbiomes) are critical for functional regulations of the body organ systems-the digestive or 'gut' microbiome being the most described so far. Based on extensive research in humans, microbiomes in the reproductive tract may play a role in reproductive functions and pregnancy. However, in wild animal species, those microbiomes have been poorly studied. Despite current technical limits of microbial identification in all animal species, there is a link between microbial communities (within reproductive systems) and fertility, from conception to birth outcome [8]. The presence of microbiota or their constituent molecules in the uterus appears to go together with healthy physiological functions. Lessons learned from the gut microbiome suggest that the microbiota of the uterus may potentially modulate immune cell subsets needed for implantation and have implications for tissue morphology. There is still a need to further investigate if a "core" or resident uterine microbiota exists and the contributions to health and homeostasis [9]. While studies on a 'baseline' microbial state of the uterus representing the optimal starting point for implantation and subsequent placenta formation are conducted in many animal models [10], similar investigations in species using embryonic diapause are now warranted.

\section{Using advanced techniques and approaches to better understand embryonic diapause}

Innovative strategies must be used to explore new players mentioned above and other understudied mechanisms.

\section{'Omics'}

Next generation sequencing and functional genomics as well as associated approaches (transcriptomics, metabolomics, proteomics, etc.) have opened a new era in biological explorations. As mentioned above, microRNAs with robust changes have been identified across multiple studies in both human and mouse by integrating transcriptomic data of mRNA and microRNA, as well as metabolomics data of naïve to primed transition in both human and mouse stem cells [3]. Recent proteomic studies in the roe deer uterus also illustrate the value of these technologies [11]. Using those cutting-edge tools will increase, for instance, our knowledge on epigenetic contributions to the interactions between the embryo and the uterus like in other species studied so far [12]. Furthermore, analysis of extracellular vesicles and non-invasive detection of circulating extra-cellular DNA (or cell-free DNA) would also be useful to explore potential markers of the different stages of the embryonic diapause and the uterine environment (similar to what has been proposed to monitor pregnancy complications [13]).

\section{$\underline{\text { In vitro culture systems and non-invasive observations }}$}

Time-lapse imaging of in vitro of embryo development would bring a lot of information like in other animal species [14]. Development of 3D culture system of endometrial cells [15] coupled with the design of organs on-a-chip [16] would also be essential to further understand embryonic diapause. Similarly, in vivo imaging of fetal development (high resolution in utero imaging of early primate embryogenesis [17]) would be of great value. Lastly, tools currently used in digital agriculture to monitor physiological status of livestock species could also be helpful. Specifically, small connected devices (placed in the digestive tract, the reproductive tract, sub-cutaneous) can closely monitor body temperature or local $\mathrm{pH}$ for accurate detection of key events like estrus or calving [18]. 


\section{Systems biology and holistic approaches}

Besides new technologies, more global analysis and interpretations of data would significantly contribute to the understanding of embryonic diapause in different species. Systems biology is an experimental and theoretical framework that treats biology as an informational science, and seeks to study the behavior of biological systems as a whole [19]. Based on the use of bioinformatics, systems biology attempts to fuse developmental biology, ecology, and physiology into an integrative science. In that context, embryonic diapause could be considered as a system itself that connects with other physiological and biological systems. Another essential aspect would be to accurately measure the influence of environmental changes (climate, pollution, nutrition, pathogens, stress) on embryonic diapause mechanisms and kinetics. Cumulative factors undoubtedly influence female physiology during the peri-conception period. During extended embryonic diapause, those factors might be even more detrimental to the embryo than in other mammalian species. These studies could be possible by following the principles of allostatic load developed in other animal species [20].

\section{Lessons from non-mammalian organisms}

Killifish and insect diapause strategies are more related to long-term biostabilization, especially in dry conditions at non-physiological temperatures. The annual killifish (Austrofundulus limnaeus) can enter into a state of metabolic and developmental dormancy during embryogenesis. Embryos may also "escape" entry into diapause and develop continuously until hatching. Phenotype appears to be influenced by maternal provisioning based on the observation that young females produce predominately escape embryos and older females produce diapausing embryos. Maternal provisioning is known in many vertebrates to direct early development prior to the initiation of embryonic gene transcription. Interestingly, embryos that are destined for either the diapause or escape phenotypes have unique profiles of mRNA isoforms at fertilization; well before they are morphologically distinct. Maternally packaged gene products could coordinate cellular events that determine developmental trajectory. In addition, expression of environmentally responsive microRNAs during development could override maternal provisioning. It is suggested that maternal programming of diapause through the packaging of specific mRNAs as well as temperature induced microRNAs that target maternal transcripts allowing an escape from the diapause trajectory. These studies not only impact the understanding of genetic mechanisms that regulate entrance into diapause but also provide insight into epigenetic control of development [21]. There might also be some common genes or similar epigenetic contributions like H3K4me3 that could help to better understand diapause in mammals [22]. New tools used in killifish studies also are inspiring. A recent publication reported the use of Fluorescent Ubiquitination-based Cell Cycle Indicator (FUCCI) to explore cell cycle dynamics during killifish development. It revealed surprising levels of coordination of cellular dynamics during diapause and provided a reference framework for further analyses of embryonic diapause [23]. 


\section{Lessons from embryonic diapause to understand other biological mechanisms and develop new treatments}

\section{Stem cell acquisition and maintenance in domestic and endangered species}

First stem cells were derived from the inner cell mass of diapause blastocysts. Further studies demonstrated in mouse embryos that Myc controls the biosynthetic machinery of stem cells without affecting their potency, thus regulating their entry and exit from the dormant state [24]. Target genes of consistently changing microRNAs in mouse embryos also regulate important metabolic and developmental pathways in naïve to primed transition [3]. Dynamics of embryonic diapause are affected by microRNA regulated stress and inflammation response genes. A recent study demonstrates that Hesx 1 is a novel factor involved in the regulation of embryonic stem cell fate. Hesx 1 also is expressed in the inner cell mass of the preimplantation mouse embryo, where it is required to resume development following diapause [25]. It was recently shown that entrance into and exit from dormant status varied depending on cell types and location of cells in the mouse embryo. These results suggest that embryonic diapause includes multiple steps and the mechanisms involved in cellular dormancy may be distinct between embryonic regions.

Entrance into and exit from dormant status varied depending on cell types and location of cells in an embryo [26]. While more studies are needed in embryonic diapause models, overall knowledge will be beneficial to the isolation and maintenance of stem cell state in other species.

\section{Cancer treatments and contraception methods}

During diapause, cell division is reversibly suspended. The reactivation of the embryo, including its rapid resumption of cell proliferation and increased metabolism followed by its subsequent implantation, has many similarities to the invasive nature of cancer cells [1]. There is a potential application of mechanisms involved in those processes in the identification of novel cancer therapies to halt or slowdown cell divisions (keeping them in a dormant stage). A perfect example of this is mTOR inhibitors inducing a paused pluripotent stage [27]. Onset of embryonic diapause could be a good model for contraception (arresting the embryo development, preventing the implantation, interfering with signals from central nervous system, manipulating the neuroendocrine system).

\section{Short/medium term storage of embryos}

Understanding embryonic diapause in mammals can provide further insight into the minimum requirements to preserve vital structures and functions of an embryo for an extended period of time at physiological temperatures (see Wauters et al. 2019 in the proceedings). This application would be convenient for embryo transfers in companion and livestock species. However, lessons from diapause and anhydrobiosis in killifish could contribute to the developmental of longer term storage strategies that could be applied to more species [28].

\section{Solving issues of implantation failure in other species}

As mentioned above, the role of bone marrow derived immune cells and preferred localizations of implantation in the uterus are intriguing [7]. While this could lead to a better understanding of the signals involved in the resumption after embryonic diapause, that process could also inform about the connection between inflammation and embryo implantation in other mammalian species. Immunological detection during early pregnancy is complex and understanding early fetal loss is key in livestock species as well as in humans [29] 


\section{Conclusions}

Current knowledge of embryonic diapause in more than 130 mammalian species allows to distinguish two main types - lactational versus seasonal controlled diapause [2]. Further understanding of embryonic diapause may reveal new mechanisms, more specificities as well as more commonalities between species. This will be possible by conducting more comparative studies (using the most advanced technologies) and integrating data into more comprehensive characterizations of phenotypes. More explorations of different biological systems (including the neuroendocrine system) related to embryonic diapause are warranted. It will also be essential to measure the influence of environmental changes on maternal physiology and the arrested embryo as well as on embryonic diapause timing and mechanisms. Overall, new directions and approaches mentioned in this review will help to define some themes for a future symposium (sooner than later!).

\section{Declaration of interest}

The author declares that there is no conflict of interest that could be perceived as prejudicing the impartiality of the research reported

\section{Funding}

N/A

\section{Acknowledgements}

Thanks to Marilyn Renfree and Bruce Murphy for constructive comments and positive feedback.

\section{References}

[1] Fenelon JC and Renfree MB. 2018 The history of the discovery of embryonic diapause in mammals. Biol Reprod 99 242-251.

[2] Renfree MB and Fenelon JC. 2017 The enigma of embryonic diapause.

[3] Wang Y, Hussein AM, Somasundaram L, Sankar R, Detraux D, Mathieu J and Ruohola-Baker H. 2019 microRNAs Regulating Human and Mouse Naïve Pluripotency. Int J Mol Sci 20.

[4] Fenelon JC, Lefèvre PL, Banerjee A and Murphy BD. 2017 Regulation of diapause in carnivores. Reprod Domest Anim 52 Suppl 2 12-17.

[5] Anuradha, Banerjee A and Krishna A. 2019 Role of putrescine in ovary and embryo development in fruit bat Cynopterus sphinx during embryonic diapause. Mol Reprod Dev.

[6] Lima PDA, Zhang J, Dunk C, Lye SJ and Anne Croy B. 2014 Leukocyte driven-decidual angiogenesis in early pregnancy. Cell Mol Immunol 11 522-537.

[7] Tal R, Shaikh S, Pallavi P, Tal A, López-Giráldez F, Lyu F, Fang Y-Y, Chinchanikar S, Liu Y, Kliman HJ, Alderman M, Pluchino N, et al. 2019 Adult bone marrow progenitors become decidual cells and contribute to embryo implantation and pregnancy. PLOS Biol 17 e3000421.

[8] Comizzoli P and Power M. 2019 Reproductive Microbiomes in Wild Animal Species: A New Dimension in Conservation Biology. 225-240.

[9] Baker JM, Chase DM and Herbst-Kralovetz MM. 2018 Uterine microbiota: Residents, tourists, or invaders? Front Immunol 9.

[10] Benner M, Ferwerda G, Joosten I and van der Molen RG. 2018 How uterine microbiota might be responsible for a receptive, fertile endometrium. Hum Reprod Update 24 393-415. 
[11] van der Weijden VA, Bick JT, Bauersachs S, Arnold GJ, Fröhlich T, Drews B and Ulbrich SE. 2019 Uterine fluid proteome changes during diapause and resumption of embryo development in roe deer (Capreolus capreolus). Reproduction 158 13-24.

[12] Kong S, Zhou C, Bao H, Ni Z, Liu M, He B, Huang L, Sun Y and Wang H, Lu J. 2019 Epigenetic control of embryo-uterine crosstalk at peri-implantation. Cell Mol Life Sci.

[13] Kone cná B, Tóthová L and Repiská G. 2019 Exosomes-associated DNA-new marker in pregnancy complications? Int J Mol Sci 20.

[14] Chitnis A and Dalle Nogare D. 2018 Time-lapse imaging beyond the diffraction limit. Methods 150 32-41.

[15] Dundon M, Madden O and Comizzoli P. 2019 Three-dimensional culture of endometrial cells from domestic cats: A new in vitro platform for assessing plastic toxicity. PLoS One 14.

[16] Mancini V and Pensabene V. 2019 Organs-on-chip models of the female reproductive system. Bioengineering 6.

[17] Meyer N, Schüler T and Zenclussen AC. 2018 High frequency ultrasound for the analysis of fetal and placental development in vivo. J Vis Exp 2018.

[18] Saint-Dizier M and Chastant-Maillard S. 2018 Potential of connected devices to optimize cattle reproduction. Theriogenology 112 53-62.

[19] Edelman LB, Chandrasekaran S and Price ND. 2010 Systems biology of embryogenesis. Reprod Fertil Dev 22 98-105.

[20] Edwards KL, Edes AN and Brown JL. 2019 Stress, well-being and reproductive success. Advances in Experimental Medicine and Biology, vol. 1200. Springer New York LLC 91-162.

[21] Wagner JT, Singh PP, Romney AL, Riggs CL, Minx P, Woll SC, Roush J, Warren WC, Brunet A and Podrabsky JE. 2018 The genome of Austrofundulus limnaeus offers insights into extreme vertebrate stress tolerance and embryonic development. BMC Genomics 19 1-21.

[22] Hu CK and Brunet A. 2018 The African turquoise killifish: A research organism to study vertebrate aging and diapause. Aging Cell 17 1-15.

[23] Dolfi L, Ripa R, Antebi A, Valenzano DR and Cellerino A. 2019 Cell cycle dynamics during diapause entry and exit in an annual killifish revealed by $\mathrm{FUCCI}$ technology. Evodevo 10.

[24] Scognamiglio R, Cabezas-Wallscheid N, Thier MC, Altamura S, Reyes A, Prendergast ÁM, Baumgärtner D, Carnevalli LS, Atzberger A, Haas S, von Paleske L, Boroviak T, et al. 2016 Myc Depletion Induces a Pluripotent Dormant State Mimicking Diapause. Cell 164 668-80.

[25] Pozzi S, Bowling S, Apps J, Brickman JM, Rodriguez TA and Martinez-Barbera JP. 2019 Genetic Deletion of Hesx1 Promotes Exit from the Pluripotent State and Impairs Developmental Diapause. Stem Cell Reports.

[26] Kamemizu C and Fujimori T. 2019 Distinct dormancy progression depending on embryonic regions during mouse embryonic diapauset. Biol Reprod 100 1204-1214.

[27] Bulut-Karslioglu A, Biechele S, Jin H, MacRae TA, Hejna M, Gertsenstein M, Song JS and RamalhoSantos M. 2016 Inhibition of mTOR induces a paused pluripotent state. Nature 540 119-123.

[28] Hand SC, Moore DS and Patil Y. 2018 Challenges during diapause and anhydrobiosis: Mitochondrial bioenergetics and desiccation tolerance. IUBMB Life 70 1251-1259.

[29] Ott TL. 2019 Symposium review: Immunological detection of the bovine conceptus during early pregnancy. J Dairy Sci 102 3766-3777. 